\title{
A Modified Mann Iteration by Boundary Point Method for Finding Minimum-Norm Fixed Point of Nonexpansive Mappings
}

\author{
Songnian $\mathrm{He}^{1,2}$ and Wenlong $\mathrm{Zhu}^{1,2}$ \\ ${ }^{1}$ College of Science, Civil Aviation University of China, Tianjin 300300, China \\ ${ }^{2}$ Tianjin Key Laboratory for Advanced Signal Processing, Civil Aviation University of China, Tianjin 300300, China \\ Correspondence should be addressed to Songnian He; hesongnian2003@yahoo.com.cn
}

Received 22 November 2012; Accepted 13 February 2013

Academic Editor: Satit Saejung

Copyright (c) 2013 S. He and W. Zhu. This is an open access article distributed under the Creative Commons Attribution License, which permits unrestricted use, distribution, and reproduction in any medium, provided the original work is properly cited.

Let $H$ be a real Hilbert space and $C \subset H$ a closed convex subset. Let $T: C \rightarrow C$ be a nonexpansive mapping with the nonempty set of fixed points $\operatorname{Fix}(T)$. Kim and Xu (2005) introduced a modified Mann iteration $x_{0}=x \in C, y_{n}=\alpha_{n} x_{n}+\left(1-\alpha_{n}\right) T x_{n}, x_{n+1}=$ $\beta_{n} u+\left(1-\beta_{n}\right) y_{n}$, where $u \in C$ is an arbitrary (but fixed) element, and $\left\{\alpha_{n}\right\}$ and $\left\{\beta_{n}\right\}$ are two sequences in $(0,1)$. In the case where $0 \in C$, the minimum-norm fixed point of $T$ can be obtained by taking $u=0$. But in the case where $0 \notin C$, this iteration process becomes invalid because $x_{n}$ may not belong to $C$. In order to overcome this weakness, we introduce a new modified Mann iteration by boundary point method (see Section 3 for details) for finding the minimum norm fixed point of $T$ and prove its strong convergence under some assumptions. Since our algorithm does not involve the computation of the metric projection $P_{C}$, which is often used so that the strong convergence is guaranteed, it is easy implementable. Our results improve and extend the results of Kim, Xu, and some others.

\section{Introduction}

Let $C$ be a subset of a real Hilbert space $H$ with an inner product and its induced norm is denoted by $\langle\cdot, \cdot\rangle$ and $\|\cdot\|$, respectively. A mapping $T: C \rightarrow C$ is called nonexpansive if $\|T x-T y\| \leq\|x-y\|$ for all $x, y \in C$. A point $x \in C$ is called a fixed point of $T$ if $T x=x$. Denote by $\operatorname{Fix}(T)=\{x \in C \mid T x=$ $x\}$ the set of fixed points of $T$. Throughout this paper, $\operatorname{Fix}(T)$ is always assumed to be nonempty.

The iteration approximation processes of nonexpansive mappings have been extensively investigated by many authors (see [1-12] and the references therein). A classical iterative scheme was introduced by Mann [13], which is defined as follows. Take an initial guess $x_{0} \in C$ arbitrarily and define $\left\{x_{n}\right\}$, recursively, by

$$
x_{n+1}=\alpha_{n} x_{n}+\left(1-\alpha_{n}\right) T x_{n}, \quad n \geq 0,
$$

where $\left\{\alpha_{n}\right\}$ is a sequence in the interval $[0,1]$. It is well known that under some certain conditions the sequence $\left\{x_{n}\right\}$ generated by (1) converges weakly to a fixed point of $T$, and
Mann iteration may fail to converge strongly even if it is in the setting of infinite-dimensional Hilbert spaces [14].

Some attempts to modify the Mann iteration method (1) so that strong convergence is guaranteed have been made. Nakajo and Takahashi [1] proposed the following modification of the Mann iteration method (1):

$$
\begin{gathered}
x_{0}=x \in C, \\
y_{n}=\alpha_{n} x_{n}+\left(1-\alpha_{n}\right) T x_{n}, \\
C_{n}=\left\{z \in C:\left\|y_{n}-z\right\| \leq\left\|x_{n}-z\right\|\right\}, \\
Q_{n}=\left\{z \in C:\left\langle x_{n}-z, x_{0}-x_{n}\right\rangle \geq 0\right\}, \\
x_{n+1}=P_{C_{n} \cap Q_{n}}\left(x_{0}\right),
\end{gathered}
$$

where $P_{K}$ denotes the metric projection from $H$ onto a closed convex subset $K$ of $H$. They proved that if the sequence $\left\{\alpha_{n}\right\}$ is bounded above from one, then $\left\{x_{n}\right\}$ defined by (2) converges strongly to $P_{\operatorname{Fix}(T)}\left(x_{0}\right)$. But, at each iteration step, an additional projection is needed to calculate, which is not easy in general. To overcome this weakness, Kim and Xu [15] proposed a simpler modification of Mann's iteration scheme, 
which generates the iteration sequence $\left\{x_{n}\right\}$ via the following formula:

$$
\begin{gathered}
x_{0}=x \in C, \\
y_{n}=\alpha_{n} x_{n}+\left(1-\alpha_{n}\right) T x_{n}, \\
x_{n+1}=\beta_{n} u+\left(1-\beta_{n}\right) y_{n},
\end{gathered}
$$

where $u \in C$ is an arbitrary (but fixed) element in $C$, and $\left\{\alpha_{n}\right\}$ and $\left\{\beta_{n}\right\}$ are two sequences in $(0,1)$. In the setting of Banach spaces, Kim and Xu proved that the sequence $\left\{x_{n}\right\}$ generated by (3) converges strongly to the fixed point $P_{\mathrm{Fix}(T)} u$ of $T$ under certain appropriate assumptions on the sequences $\left\{\alpha_{n}\right\}$ and $\left\{\beta_{n}\right\}$.

In many practical problems, such as optimization problems, finding the minimum norm fixed point of nonexpansive mappings is quite important. In the case where $0 \in C$, taking $u=0$ in (3), the sequence $\left\{x_{n}\right\}$ generated by (3) converges strongly to the minimum norm fixed point of $T$ [15]. But, in the case where $0 \notin C$, the iteration scheme (3) becomes invalid because $x_{n}$ may not belong to $C$.

To overcome this weakness, a natural way to modify algorithm (3) is adopting the metric projection $P_{C}$ so that the iteration sequence belongs to $C$; that is, one may consider the scheme as follows:

$$
\begin{gathered}
x_{0}=x \in C, \\
y_{n}=\alpha_{n} x_{n}+\left(1-\alpha_{n}\right) T x_{n}, \\
x_{n+1}=P_{C}\left(\beta_{n} u+\left(1-\beta_{n}\right) y_{n}\right) .
\end{gathered}
$$

However, since the computation of a projection onto a closed convex subset is generally difficult, algorithm (4) may not be a well choice.

The main purpose of this paper is to introduce a new modified Mann iteration for finding the minimum norm fixed point of $T$, which not only has strong convergence under some assumptions but also has nothing to do with any projection operators. At each iteration step, a point in $\partial C$ (the boundary of $C$ ) is determined via a particular way, so our modification method is called boundary point method (see Section 3 for details). Moreover, since our algorithm does not involve the computation of the metric projection, it is very easy implementable.

The rest of this paper is organized as follows. Some useful lemmas are listed in the next section. In the last section, a function defined on $C$ is given firstly, which is important for us to construct our algorithm, then our algorithm is introduced and the strong convergence theorem is proved.

\section{Preliminaries}

Throughout this paper, we adopt the notations listed as follows:

(1) $x_{n} \rightarrow x:\left\{x_{n}\right\}$ converges strongly to $x$;

(2) $x_{n} \rightarrow x:\left\{x_{n}\right\}$ converges weakly to $x$;

(3) $\omega_{w}\left(x_{n}\right)$ denotes the set of cluster points of $\left\{x_{n}\right\}$ (i.e., $\omega_{w}\left(x_{n}\right)=\left\{x: \exists\left\{x_{n_{k}}\right\} \subset\left\{x_{n}\right\}\right.$ such that $\left.\left.x_{n_{k}} \rightarrow x\right\}\right)$;

(4) $\partial C$ denotes the boundary of $C$.
We need some lemmas and facts listed as follows.

Lemma 1 (see [16]). Let $K$ be a closed convex subset of a real Hilbert space $H$ and let $P_{K}$ be the (metric of nearest point) projection from $H$ onto $K$ (i.e., for $x \in H, P_{K} x$ is the only point in $K$ such that $\left.\left\|x-P_{K} x\right\|=\inf \{\|x-z\|: z \in K\}\right)$. Given $x \in H$ and $z \in K$. Then $z=P_{K} x$ if and only if there holds the following relation:

$$
\langle x-z, y-z\rangle \leq 0, \quad \forall y \in K .
$$

Since $\operatorname{Fix}(T)$ is a closed convex subset of a real Hilbert space $H$, so the metric projection $P_{\mathrm{Fix}(T)}$ is reasonable and thus there exists a unique element, which is denoted by $x^{\dagger}$, in $\operatorname{Fix}(T)$ such that $\left\|x^{\dagger}\right\|=\inf _{x \in \operatorname{Fix}(T)}\|x\|$; that is, $x^{\dagger}=P_{\operatorname{Fix}(T)} 0$. $x^{\dagger}$ is called the minimum norm fixed point of $T$.

Lemma 2 (see [17]). Let $H$ be a real Hilbert space. Then there holds the following well-known results:

(G1) $\|x-y\|^{2}=\|x\|^{2}-2\langle x, y\rangle+\|y\|^{2}$ for all $x, y \in H$;

(G2) $\|x+y\|^{2} \leq\|x\|^{2}+2\langle y, x+y\rangle$ for all $x, y \in H$.

We will give a definition in order to introduce the next lemma. A set $C \subset H$ is weakly closed if for any sequence $\left\{x_{n}\right\} \subset$ $C$ such that $x_{n} \rightarrow x$, there holds $x \in C$.

Lemma 3 (see $[18,19]$ ). If $C \subset H$ is convex, then $C$ is weakly closed if and only if $C$ is closed.

Assume $C \subset H$ is weakly closed; a function $f: C \rightarrow$ $\mathbb{R}^{1}$ is called weakly lower semicontinuous at $x_{0} \in C$ if for any sequence $\left\{x_{n}\right\} \subset C$ such that $x_{n} \rightarrow x$; then $f(x) \leq$ $\lim \inf _{n \rightarrow \infty} f\left(x_{n}\right)$ holds. Generally, we called $f$ weakly lower semi-continuous over $C$ if it is weakly lower semi-continuous at each point in $C$.

Lemma 4 (see $[18,19])$. Let $C$ be a subset of a real Hilbert space $H$ and let $f: C \rightarrow \mathbb{R}^{1}$ be a real function; then $f$ is weakly lower semi-continuous over $C$ if and only if the set $\{x \in$ $C \mid f(x) \leq a\}$ is weakly closed subset of $H$, for any $a \in \mathbb{R}^{1}$.

Lemma 5 (see [20]). Let $C$ be a closed convex subset of a real Hilbert space $H$ and let $T: C \rightarrow C$ be a nonexpansive mapping such that $\operatorname{Fix}(T) \neq \emptyset$. If a sequence $\left\{x_{n}\right\}$ in $C$ is such that $x_{n} \rightarrow z$ and $\left\|x_{n}-T x_{n}\right\| \rightarrow 0$, then $z=T z$.

The following is a sufficient condition for a real sequence to converge to zero.

Lemma 6 (see $[21,22]$ ). Let $\left\{\alpha_{n}\right\}$ be a nonnegative real sequence satisfying

$$
\alpha_{n+1} \leq\left(1-\gamma_{n}\right) \alpha_{n}+\gamma_{n} \delta_{n}+\sigma_{n}, \quad n=0,1,2 \ldots
$$

If $\left\{\gamma_{n}\right\}_{n=1}^{\infty} \subset(0,1),\left\{\delta_{n}\right\}_{n=1}^{\infty}$ and $\left\{\sigma_{n}\right\}_{n=1}^{\infty}$ satisfy the conditions:

(A1) $\sum_{n=1}^{\infty} \gamma_{n}=\infty$;

(A2) either $\lim \sup _{n \rightarrow \infty} \delta_{n} \leq 0$ or $\sum_{n=1}^{\infty}\left|\gamma_{n} \delta_{n}\right|<\infty$;

(A3) $\sum_{n=1}^{\infty}\left|\sigma_{n}\right|<\infty$;

then $\lim _{n \rightarrow \infty} \alpha_{n}=0$. 


\section{Iterative Algorithm}

Let $C$ be a closed convex subset of a real Hilbert space $H$. In order to give our main results, we first introduce a function $h: C \rightarrow[0,1]$ by the following definition:

$$
h(x)=\inf \{\lambda \in[0,1] \mid \lambda x \in C\}, \quad \forall x \in C .
$$

Since $C$ is closed and convex, it is easy to see that $h$ is well defined. Obviously, $h(x)=0$ for all $x \in C$ in the case where $0 \in C$. In the case where $0 \notin C$, it is also easy to see that $h(x) x \in \partial C$ and $h(x)>0$ for every $x \in C$ (otherwise, $h(x)=$ 0 ; we have $0 \in C$; this is a contradiction).

An important property of $h(x)$ is given as follows.

Lemma 7. $h(x)$ is weakly lower semi-continuous over C.

Proof. If $0 \in C$, then $h(x)=0$ for all $x \in C$ and the conclusion is clear. For the case $0 \notin C$, using Lemma 4 , in order to show that $h(x)$ is weakly lower semi-continuous, it suffices to verify that

$$
C_{a}^{-}=\{x \in C \mid h(x) \leq a\}
$$

is a weakly closed subset of $H$ for every $a \in \mathbb{R}^{1}$; that is, if $\left\{x_{n}\right\} \subset C_{a}^{-}$such that $x_{n} \rightarrow x$, then $x \in C_{a}^{-}$(i.e., $h(x) \leq$ a). Without loss of generality, we assume that $0<a<1$ (otherwise, there hold $C_{a}^{-}=C$ for $a \geq 1$ and $C_{a}^{-}=\emptyset$ for $a \leq 0$, resp., and the conclusion holds obviously). Noting $C$ is convex, we have from the definition of $h(x)$ that for each $\lambda \in[a, 1], \lambda x_{n} \in C$ holds for all $n \geq 1$. Clearly, $\lambda x_{n}-\lambda x$. Using Lemma 3 , then $\lambda x \in C$. This implies that

$$
[a, 1] \subset\{\lambda \in(0,1] \mid \lambda x \in C\} .
$$

Consequently,

$$
h(x)=\inf \{\lambda \in(0,1] \mid \lambda x \in C\} \leq a
$$

and this completes the proof.

Since the function $h(x)$ will be important for us to construct the algorithm of this paper below, it is necessary to explain how to calculate $h(x)$ for any given $x \in C$ in actual computing programs. In fact, in practical problem, $C$ is often a level set of a convex function $c$; that is, $C$ is of the form $C=\{x \in H \mid c(x) \leq r\}$, where $r$ is a real constant. Without loss of generality, we assume that

$$
C=\{x \in H \mid c(x) \leq 0\}
$$

and $0 \notin C$. Then it is easy to see that, for a given $x \in C$, we have

$$
h(x)=\inf \{\lambda \in(0,1] \mid c(\lambda x)=0\} .
$$

Thus, in order to get the value $h(x)$, we only need to solve a algebraic equation with a single variable $\lambda$, which can be solved easily using many methods, for example, dichotomy method on the interval $[0,1]$. In general, solving a algebraic equation above is quite easier than calculating the metric projection $P_{C}$. To illustrate this viewpoint, we give the following simple example.
Example 8. Let $A: H \rightarrow H$ be a strongly positive linear bounded operator with coefficient $r$; that is, there is a constant $r>0$ with the property $\langle A x, x\rangle \geq r\|x\|^{2}$, for all $x \in H$. Define a convex function $\varphi: H \rightarrow \mathbb{R}^{1}$ by

$$
\varphi(x)=\langle A x, x\rangle-3\langle x, u\rangle+\left\langle A x^{*}, x^{*}\right\rangle, \quad \forall x \in H,
$$

where $u \neq 0$ is a given point in $H$ and $x^{*}$ is the only solution of the equation $A x=u$. (Notice that $A$ is a monogamy.) Setting $C=\{x \in H: \varphi(x) \leq 0\}$, then it is easy to show that $C$ is a nonempty convex closed subset of $H$ such that $0 \notin C$. (Note that $\varphi\left(x^{*}\right)=-\left\langle A x^{*}, x^{*}\right\rangle<0$ and $\varphi(0)=\left\langle A x^{*}, x^{*}\right\rangle>0$.) For a given $x \in C$, we have $\varphi(x) \leq 0$. In order to get $h(x)$, let $\varphi(\lambda x)=0$, where $\lambda \in(0,1]$ is an unknown number. Thus we obtain an algebraic equation

$$
\langle A x, x\rangle \lambda^{2}-3\langle x, u\rangle \lambda+\left\langle A x^{*}, x^{*}\right\rangle=0 .
$$

Consequently, we have

$$
\begin{aligned}
\lambda & =\frac{3\langle x, u\rangle-\sqrt{9\langle x, u\rangle^{2}-4\langle A x, x\rangle\left\langle A x^{*}, x^{*}\right\rangle}}{2\langle A x, x\rangle} \\
& =\frac{2\left\langle A x^{*}, x^{*}\right\rangle}{3\langle x, u\rangle+\sqrt{9\langle x, u\rangle^{2}-4\langle A x, x\rangle\left\langle A x^{*}, x^{*}\right\rangle}},
\end{aligned}
$$

that is,

$$
h(x)=\frac{2\left\langle A x^{*}, x^{*}\right\rangle}{3\langle x, u\rangle+\sqrt{9\langle x, u\rangle^{2}-4\langle A x, x\rangle\left\langle A x^{*}, x^{*}\right\rangle}} .
$$

Now we give a new modified Mann iteration by boundary point method.

Algorithm 9. Define $\left\{x_{n}\right\}$ in the following way:

$$
\begin{gathered}
x_{0}=x \in C, \\
y_{n}=\alpha_{n} x_{n}+\left(1-\alpha_{n}\right) T x_{n}, \\
x_{n+1}=\alpha_{n} \lambda_{n} x_{n}+\left(1-\alpha_{n}\right) y_{n},
\end{gathered}
$$

where $\left\{\alpha_{n}\right\} \subset(0,1)$ and $\lambda_{n}=\max \left\{\lambda_{n-1}, h\left(x_{n}\right)\right\}, n=0,1$, $2, \ldots$...

Since $C$ is closed and convex, we assert by the definition of $h$ that, for any given $x \in C, \beta x \in C$ holds for every $\beta \in$ $[h(x) x, 1]$, and then $\left(x_{n}\right) \subset C$ is guaranteed, where $\left(x_{n}\right)$ is generated by Algorithm 9. Obviously, $\lambda_{n}=0$ for all $n \geq 0$ if $0 \in C$. If $0 \notin C$, calculating the value $h\left(x_{n}\right)$ implies determining $h\left(x_{n}\right) x_{n}$, a boundary point of $C$, and thus our algorithm is called boundary point method.

Theorem 10. Assume that $\left\{\alpha_{n}\right\}$ and $\left\{\lambda_{n}\right\}$ satisfy the following conditions:
(D1) $\alpha_{n} /\left(1-\lambda_{n}\right) \rightarrow 0$;
(D2) $\sum_{n=1}^{\infty} \alpha_{n}\left(1-\lambda_{n}\right)=\infty$;
(D3) $\sum_{n=1}^{\infty}\left|\alpha_{n}-\alpha_{n-1}\right|<\infty$.

Then $\left\{x_{n}\right\}$ generated by (17) converges strongly to $x^{\dagger}=$ $P_{\text {Fix }(T)} 0$. 
Proof. We first show that $\left\{x_{n}\right\}$ is bounded. Taking $p \in \operatorname{Fix}(T)$ arbitrarily, we have

$$
\begin{aligned}
\left\|x_{n+1}-p\right\|= & \| \alpha_{n} \lambda_{n}\left(x_{n}-p\right)+\alpha_{n}\left(1-\alpha_{n}\right)\left(x_{n}-p\right) \\
& +\left(1-\alpha_{n}\right)^{2}\left(T x_{n}-p\right)-\alpha_{n}\left(1-\lambda_{n}\right) p \| \\
\leq & \left(\alpha_{n} \lambda_{n}+\alpha_{n}-\alpha_{n}^{2}\right)\left\|x_{n}-p\right\| \\
& +\left(1-\alpha_{n}\right)^{2}\left\|T x_{n}-p\right\| \\
& +\alpha_{n}\left(1-\lambda_{n}\right)\|p\| \\
\leq & {\left[1-\alpha_{n}\left(1-\lambda_{n}\right)\right]\left\|x_{n}-p\right\| } \\
& +\alpha_{n}\left(1-\lambda_{n}\right)\|p\| \\
\leq & \max \left\{\left\|x_{n}-p\right\|,\|p\|\right\} .
\end{aligned}
$$

By induction,

$$
\left\|x_{n}-p\right\| \leq \max \left\{\left\|x_{0}-p\right\|,\|p\|\right\}, \quad n \geq 0 .
$$

Thus, $\left\{x_{n}\right\}$ is bounded and so are $\left\{T x_{n}\right\}$ and $\left\{y_{n}\right\}$. As a result, we obtain by condition (D1) that

$$
\begin{aligned}
\left\|x_{n+1}-y_{n}\right\| & =\left\|\alpha_{n} \lambda_{n} x_{n}+\left(1-\alpha_{n}\right) y_{n}-y_{n}\right\| \\
& \leq \alpha_{n}\left(\lambda_{n}\left\|x_{n}\right\|+\left\|y_{n}\right\|\right) \longrightarrow 0, \\
\left\|y_{n}-T x_{n}\right\| & =\left\|\alpha_{n} x_{n}+\left(1-\alpha_{n}\right) T x_{n}-T x_{n}\right\| \\
& \leq \alpha_{n}\left(\left\|x_{n}\right\|+\left\|T x_{n}\right\|\right) \longrightarrow 0 .
\end{aligned}
$$

$$
\begin{aligned}
& +\left|\alpha_{n}-\alpha_{n-1}\right|\left(\left\|y_{n-1}\right\|+\lambda_{n-1}\left\|x_{n-1}\right\|\right) \\
& +\alpha_{n}\left|\lambda_{n}-\lambda_{n-1}\right|\left\|x_{n-1}\right\|, \\
\left\|y_{n}-y_{n-1}\right\|= & \| \alpha_{n} x_{n}+\left(1-\alpha_{n}\right) T x_{n} \\
& \quad-\left[\alpha_{n-1} x_{n-1}+\left(1-\alpha_{n-1}\right) T x_{n-1}\right] \| \\
= & \|\left(1-\alpha_{n}\right)\left(T x_{n}-T x_{n-1}\right)-\left(\alpha_{n}-\alpha_{n-1}\right) T x_{n-1} \\
& +\alpha_{n}\left(x_{n}-x_{n-1}\right)+\left(\alpha_{n}-\alpha_{n-1}\right) x_{n-1} \| \\
\leq & \left(1-\alpha_{n}\right)\left\|x_{n}-x_{n-1}\right\|+\left|\alpha_{n}-\alpha_{n-1}\right|\left\|T x_{n-1}\right\| \\
& +\alpha_{n}\left\|x_{n}-x_{n-1}\right\|+\left|\alpha_{n}-\alpha_{n-1}\right|\left\|x_{n-1}\right\| \\
= & \left\|x_{n}-x_{n-1}\right\|+\left|\alpha_{n}-\alpha_{n-1}\right|\left(\left\|T x_{n-1}\right\|+\left\|x_{n-1}\right\|\right) .
\end{aligned}
$$

Substituting (24) into (23), we obtain

$$
\begin{aligned}
\left\|x_{n+1}-x_{n}\right\| \leq & \left(1-\alpha_{n}\left(1-\lambda_{n}\right)\right)\left\|x_{n}-x_{n-1}\right\|+\left|\alpha_{n}-\alpha_{n-1}\right| \\
\times & \left\{\left\|y_{n-1}\right\|+\lambda_{n-1}\left\|x_{n-1}\right\|+\left(1-\alpha_{n}\right)\right. \\
& \left.\times\left(\left\|T x_{n-1}\right\|+\left\|x_{n-1}\right\|\right)\right\} \\
& +\alpha_{n}\left|\lambda_{n}-\lambda_{n-1}\right|\left\|x_{n-1}\right\| .
\end{aligned}
$$

Note the fact that $\sum_{n=1}^{\infty}\left|\lambda_{n}-\lambda_{n-1}\right|<\infty$ (since $\left(\lambda_{n}\right) \subset$ $[0,1]$ is monotone increasing) and conditions (D1)-(D3); it concludes by using Lemma 6 that $\left\|x_{n+1}-x_{n}\right\| \rightarrow 0$. Noting (20) and (25), we obtain

$$
\begin{gathered}
\left\|x_{n}-T x_{n}\right\| \leq\left\|x_{n+1}-x_{n}\right\|+\left\|x_{n+1}-y_{n}\right\| \\
+\left\|y_{n}-T x_{n}\right\| \longrightarrow 0 .
\end{gathered}
$$

Using Lemma 5, it derives that $\omega_{w}\left(x_{n}\right) \subset \operatorname{Fix}(T)$.

Then we show that

$$
\limsup _{n \rightarrow \infty}\left\langle-x^{\dagger}, x_{n+1}-x^{\dagger}\right\rangle \leq 0 .
$$

It suffices to show that

$$
\left\|x_{n+1}-x_{n}\right\| \longrightarrow 0 .
$$

Indeed take a subsequence $\left\{x_{n_{k}}\right\}$ of $\left\{x_{n}\right\}$ such that

$$
\limsup _{n \rightarrow \infty}\left\langle-x^{\dagger}, x_{n}-x^{\dagger}\right\rangle=\lim _{k \rightarrow \infty}\left\langle-x^{\dagger}, x_{n_{k}}-x^{\dagger}\right\rangle \text {. }
$$


Without loss of generality, we may assume that $x_{n_{k}} \rightarrow \bar{x}$. Noticing $x^{\dagger}=P_{\mathrm{Fix}(T)} 0$, we obtain from $\bar{x} \in \operatorname{Fix}(T)$ and Lemma 1 that

$$
\begin{aligned}
& \underset{n \rightarrow \infty}{\lim \sup }\left\langle-x^{\dagger}, x_{n}-x^{\dagger}\right\rangle \\
& \quad=\lim _{k \rightarrow \infty}\left\langle-x^{\dagger}, x_{n_{k}}-x^{\dagger}\right\rangle=\left\langle-x^{\dagger}, \bar{x}-x^{\dagger}\right\rangle \leq 0 .
\end{aligned}
$$

Finally, we show that $\left\|x_{n}-x^{\dagger}\right\| \rightarrow 0$. Using Lemma 2 and (17), it is easy to verify that

$$
\begin{aligned}
\left\|x_{n+1}-x^{\dagger}\right\|^{2}= & \left\|\alpha_{n} \lambda_{n} x_{n}+\left(1-\alpha_{n}\right) y_{n}-x^{\dagger}\right\|^{2} \\
= & \left\|\alpha_{n}\left(\lambda_{n} x_{n}-x^{\dagger}\right)+\left(1-\alpha_{n}\right)\left(y_{n}-x^{\dagger}\right)\right\|^{2} \\
\leq & \left(1-\alpha_{n}\right)^{2}\left\|y_{n}-x^{\dagger}\right\|^{2} \\
& +2 \alpha_{n}\left\langle\lambda_{n} x_{n}-x^{\dagger}, x_{n+1}-x^{\dagger}\right\rangle \\
\leq & \left(1-\alpha_{n}\right)^{2}\left\|\alpha_{n} x_{n}+\left(1-\alpha_{n}\right) T x_{n}-x^{\dagger}\right\|^{2} \\
& +2 \alpha_{n} \lambda_{n}\left\langle x_{n}-x^{\dagger}, x_{n+1}-x^{\dagger}\right\rangle \\
& +2 \alpha_{n}\left(1-\lambda_{n}\right)\left\langle-x^{\dagger}, x_{n+1}-x^{\dagger}\right\rangle \\
\leq & \left(1-\alpha_{n}\right)^{2}\left\|x_{n}-x^{\dagger}\right\|^{2}+2 \alpha_{n} \lambda_{n}\left\|x_{n}-x^{\dagger}\right\| \\
& \times\left\|x_{n+1}-x^{\dagger}\right\| \\
& +2 \alpha_{n}\left(1-\lambda_{n}\right)\left\langle-x^{\dagger}, x_{n+1}-x^{\dagger}\right\rangle .
\end{aligned}
$$

Hence,

$$
\left\|x_{n+1}-x^{\dagger}\right\|^{2} \leq\left(1-\gamma_{n}\right)\left\|x_{n}-x^{\dagger}\right\|^{2}+\gamma_{n} \sigma_{n}
$$

where

$$
\begin{gathered}
\gamma_{n}=\alpha_{n} \frac{2\left(1-\lambda_{n}\right)-\alpha_{n}}{1-\alpha_{n} \lambda_{n}}, \\
\sigma_{n}=\frac{2\left(1-\lambda_{n}\right)}{2\left(1-\lambda_{n}\right)-\alpha_{n}}\left\langle-x^{\dagger}, x_{n+1}-x^{\dagger}\right\rangle .
\end{gathered}
$$

It is not hard to prove that $\gamma_{n} \rightarrow 0, \sum_{n=0}^{\infty} \gamma_{n}=\infty$ by conditions (D1) and (D2), and $\lim \sup _{n \rightarrow \infty} \sigma_{n} \leq 0$ by (29). By Lemma 6, we concludes that $x_{n} \rightarrow x^{\dagger}$, and the proof is finished.

Finally, we point out that a more general algorithm can be given for calculating the fixed point $P_{\mathrm{Fix}(T)} u$ for any given $u \in$ $H$. In fact, it suffices to modify the definition of the function $h$ by the following form:

$$
h(x)=\inf \{\lambda \in[0,1] \mid(1-\lambda) u+\lambda x \in C\}, \quad \forall x \in C .
$$

Algorithm 11. Define $\left\{x_{n}\right\}$ in the following way:

$$
\begin{gathered}
x_{0}=x \in C, \\
y_{n}=\alpha_{n} x_{n}+\left(1-\alpha_{n}\right) T x_{n}, \\
x_{n+1}=\alpha_{n}\left(\left(1-\lambda_{n}\right) u+\lambda_{n} x_{n}\right)+\left(1-\alpha_{n}\right) y_{n},
\end{gathered}
$$

where $\left\{\alpha_{n}\right\} \subset(0,1)$ and $\lambda_{n}=\max \left\{\lambda_{n-1}, h\left(x_{n}\right)\right\}(n=0,1$, $2, \ldots)$, where $h$ is defined by (33).

By an argument similar to the proof of Theorem 10, it is easy to obtain the result below.

Theorem 12. Assume that $u \notin C$, and $\left\{\alpha_{n}\right\}$ and $\left\{\lambda_{n}\right\}$ satisfy the same conditions as in Theorem 10; then $\left\{x_{n}\right\}$ generated by (34) converges strongly to $x^{*}=P_{\operatorname{Fix}(T)} u$.

\section{Acknowledgments}

This work was supported in part by the Fundamental Research Funds for the Central Universities (ZXH2012K001) and in part by the Foundation of Tianjin Key Lab for Advanced Signal Processing.

\section{References}

[1] K. Nakajo and W. Takahashi, "Strong convergence theorems for nonexpansive mappings and nonexpansive semigroups," Journal of Mathematical Analysis and Applications, vol. 279, no. 2, pp. 372-379, 2003.

[2] J. S. Jung, "Iterative approaches to common fixed points of nonexpansive mappings in Banach spaces," Journal of Mathematical Analysis and Applications, vol. 302, no. 2, pp. 509-520, 2005.

[3] H. H. Bauschke, "The approximation of fixed points of compositions of nonexpansive mappings in Hilbert space," Journal of Mathematical Analysis and Applications, vol. 202, no. 1, pp. 150159, 1996.

[4] S. S. Chang, "Viscosity approximation methods for a finite family of nonexpansive mappings in Banach spaces," Journal of Mathematical Analysis and Applications, vol. 323, no. 2, pp. 1402-1416, 2006.

[5] F. E. Browder and W. V. Petryshyn, "Construction of fixed points of nonlinear mappings in Hilbert space," Journal of Mathematical Analysis and Applications, vol. 20, pp. 197-228, 1967.

[6] G. Marino and H. K. Xu, "Weak and strong convergence theorems for strict pseudo-contractions in Hilbert spaces," Journal of Mathematical Analysis and Applications, vol. 329, no. 1, pp. 336-346, 2007.

[7] G. Marino and H. K. Xu, "Convergence of generalized proximal point algorithms," Communications on Pure and Applied Analysis, vol. 3, no. 4, pp. 791-808, 2004.

[8] A. Moudafi, "Viscosity approximation methods for fixed-points problems," Journal of Mathematical Analysis and Applications, vol. 241, no. 1, pp. 46-55, 2000.

[9] B. Halpern, "Fixed points of nonexpanding maps," Bulletin of the American Mathematical Society, vol. 73, pp. 957-961, 1967.

[10] S. Ishikawa, "Fixed points by a new iteration method," Proceedings of the American Mathematical Society, vol. 44, pp. 147-150, 1974. 
[11] S. Reich, "Weak convergence theorems for nonexpansive mappings in Banach spaces," Journal of Mathematical Analysis and Applications, vol. 67, no. 2, pp. 274-276, 1979.

[12] N. Shioji and W. Takahashi, "Strong convergence of approximated sequences for nonexpansive mappings in Banach spaces," Proceedings of the American Mathematical Society, vol. 125, no. 12, pp. 3641-3645, 1997.

[13] W. R. Mann, "Mean value methods in iteration," Proceedings of the American Mathematical Society, vol. 4, pp. 506-510, 1953.

[14] A. Genel and J. Lindenstrauss, "An example concerning fixed points," Israel Journal of Mathematics, vol. 22, no. 1, pp. 81-86, 1975.

[15] T. H. Kim and H. K. Xu, "Strong convergence of modified Mann iterations," Nonlinear Analysis: Theory, Methods \& Applications, vol. 61, no. 1-2, pp. 51-60, 2005.

[16] C. Martinez-Yanes and H. K. Xu, "Strong convergence of the CQ method for fixed point iteration processes," Nonlinear Analysis: Theory, Methods \& Applications, vol. 64, no. 11, pp. 2400-2411, 2006.

[17] M. Li and Y. Yao, "Strong convergence of an iterative algorithm for $\lambda$-strictly pseudo-contractive mappings in Hilbert spaces," Analele stiintifice ale Universitatii Ovidius Constanta, vol. 18, no. 1, pp. 219-228, 2010.

[18] B. Beauzamy, Introduction to Banach Spaces and Their Geometry, vol. 68 of North-Holland Mathematics Studies, NorthHolland, Amsterdam, The Netherlands, 1982.

[19] J. Diestel, Geometry of Banach Spaces-Selected Topics, vol. 485 of Lecture Notes in Mathematics, Springer, Berlin, Germany, 1975.

[20] K. Goebel and W. A. Kirk, Topics in Metric Fixed Point Theory, vol. 28 of Cambridge Studies in Advanced Mathematics, Cambridge University Press, Cambridge, UK, 1990.

[21] F. Wang and H. K. Xu, "Approximating curve and strong convergence of the CQ algorithm for the split feasibility problem," Journal of Inequalities and Applications, vol. 2010, Article ID 102085, 13 pages, 2010.

[22] L. S. Liu, "Ishikawa and Mann iterative process with errors for nonlinear strongly accretive mappings in Banach spaces," Journal of Mathematical Analysis and Applications, vol. 194, no. 1, pp. 114-125, 1995. 


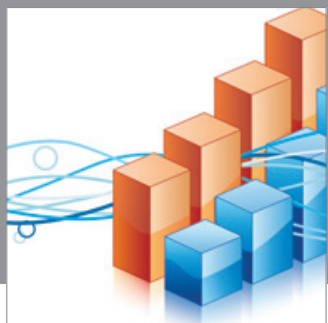

Advances in

Operations Research

mansans

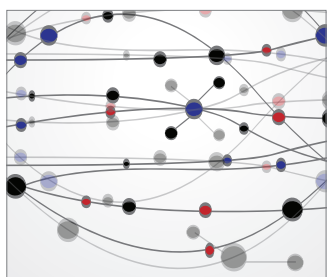

The Scientific World Journal
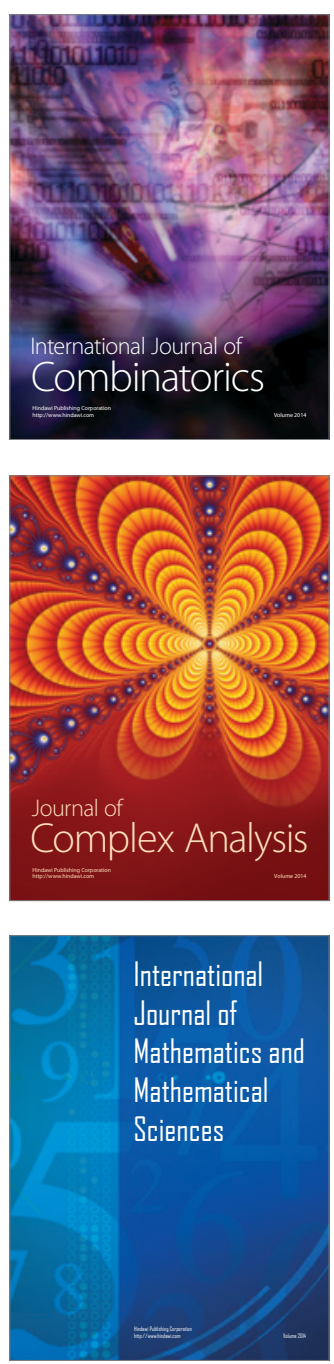
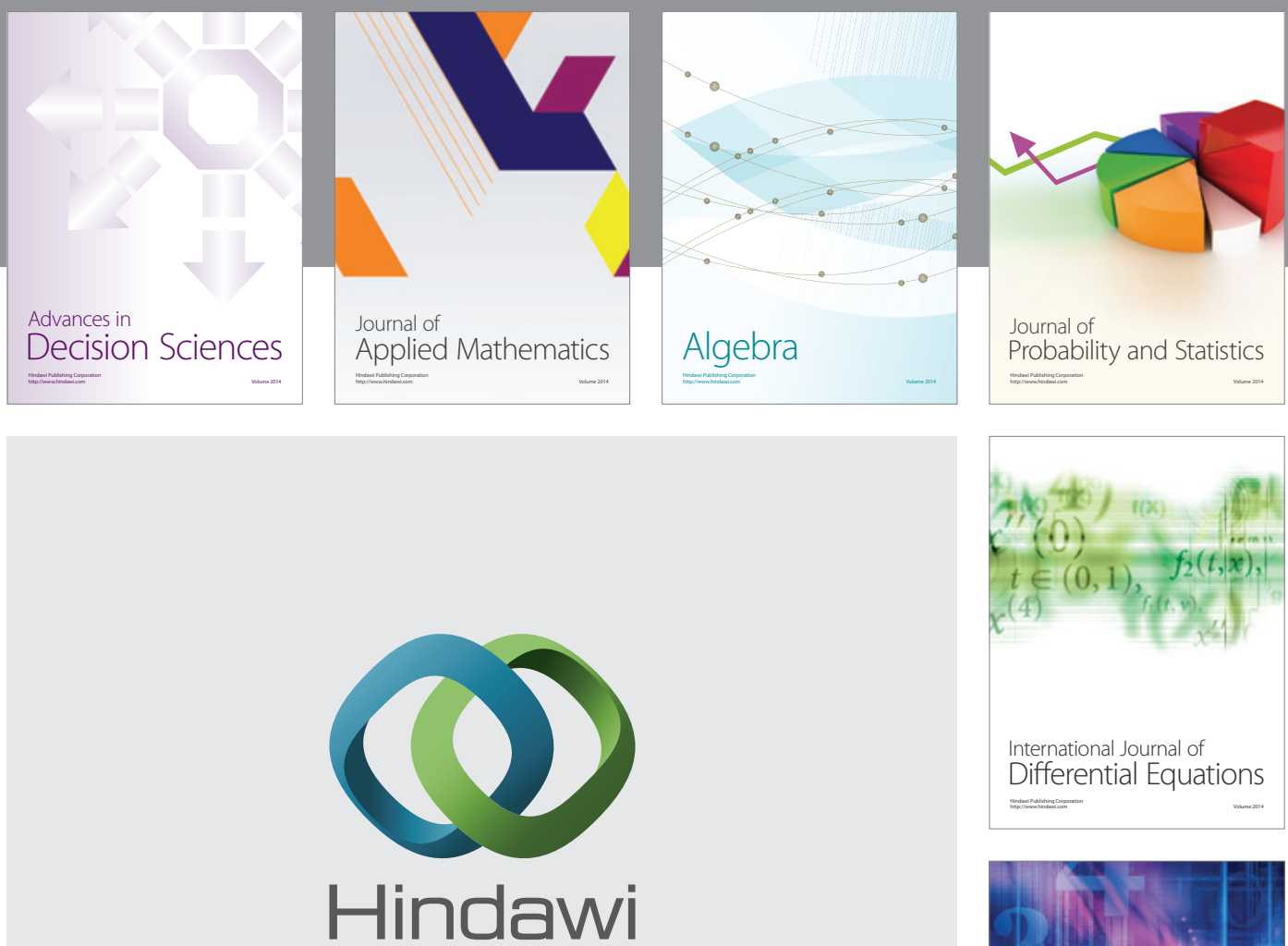

Submit your manuscripts at http://www.hindawi.com
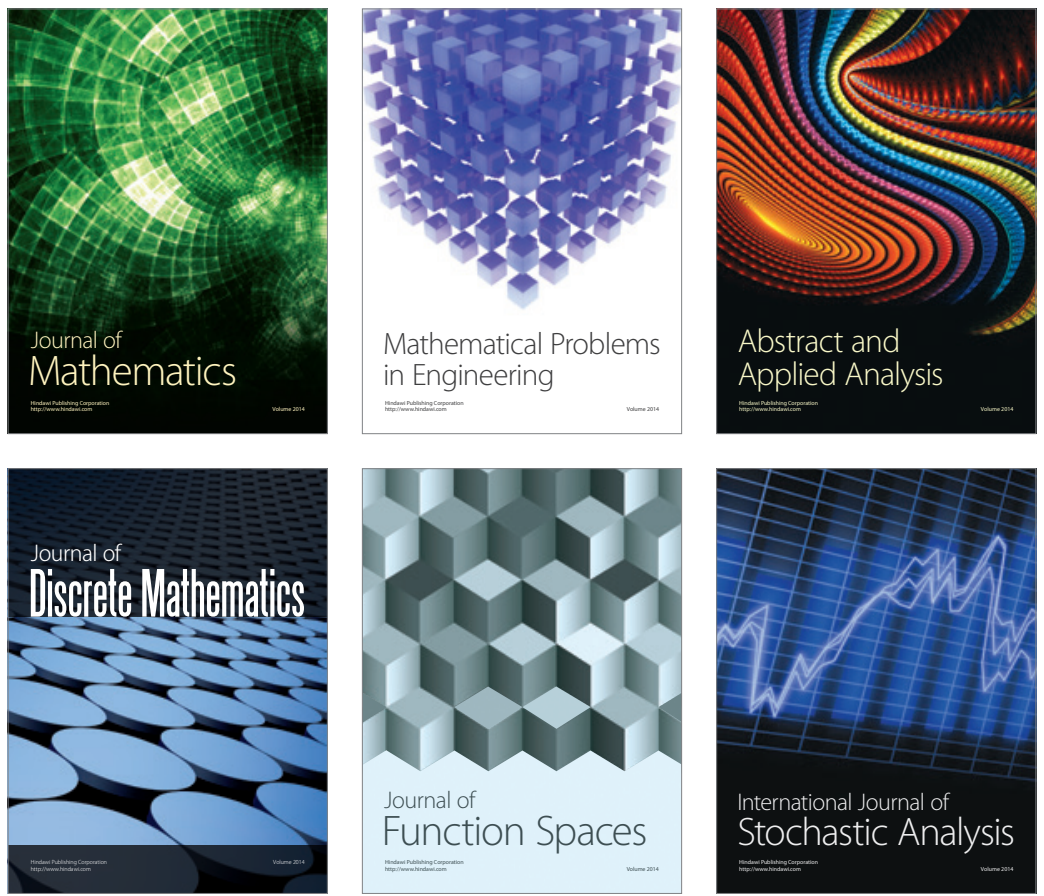

Journal of

Function Spaces

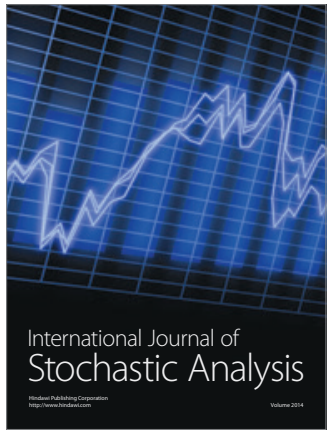

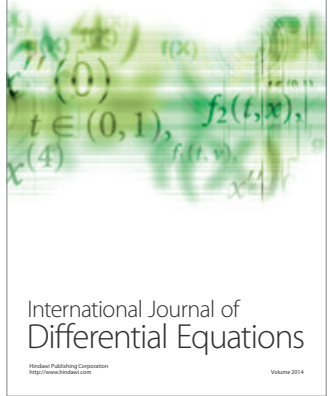
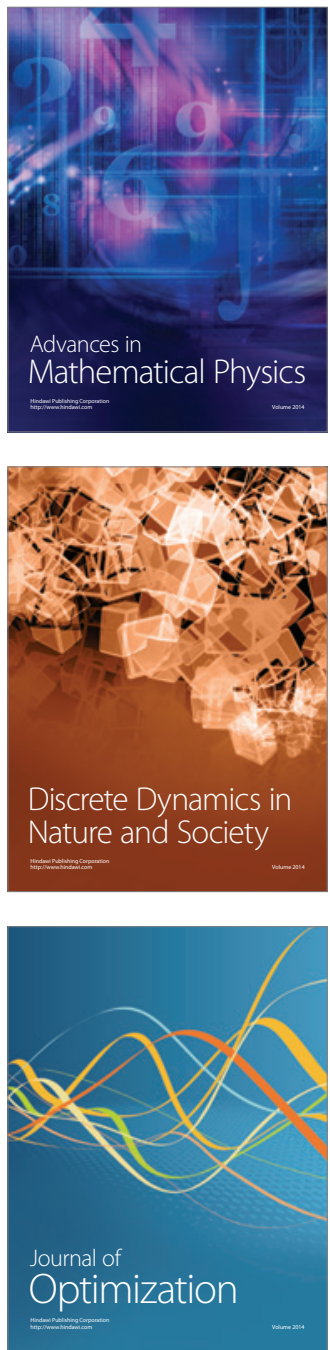